ANNALES

POLONICI MATHEMATICI

$94.3(2008)$

\title{
On the Łojasiewicz exponent at infinity of real polynomials
}

\author{
by HÀ HuY Vui (Hanoi) and Phạm Tiến So’N (Dalat)
}

\begin{abstract}
Let $f: \mathbb{R}^{n} \rightarrow \mathbb{R}$ be a nonconstant polynomial function. Using the information from the "curve of tangency" of $f$, we provide a method to determine the Łojasiewicz exponent at infinity of $f$. As a corollary, we give a computational criterion to decide if the Łojasiewicz exponent at infinity is finite or not. Then we obtain a formula to calculate the set of points at which the polynomial $f$ is not proper. Moreover, a relation between the Łojasiewicz exponent at infinity of $f$ and the problem of computing the global optimum of $f$ is also established.
\end{abstract}

1. Introduction. Let $F:=\left(f_{1}, \ldots, f_{k}\right): \mathbb{K}^{n} \rightarrow \mathbb{K}^{k}$ be a polynomial mapping, where $\mathbb{K}:=\mathbb{R}$ or $\mathbb{K}:=\mathbb{C}$. We define the Eojasiewicz exponent at infinity $\mathcal{L}_{\infty}(F)$ of the mapping $F$ to be the least upper bound of the set of all real numbers $l$ which satisfy the condition: there exist positive constants $c, r$ such that

$$
\|F(x)\| \geq c\|x\|^{l} \quad \text { for }\|x\| \geq r .
$$

If there are no such $l$, we put $\mathcal{L}_{\infty}(F):=-\infty$.

The Łojasiewicz exponent at infinity is of fundamental importance in singularity theory. In a natural way, a fundamental question arises:

- How to determine the Lojasiewicz exponent at infinity $\mathcal{L}_{\infty}(F)$ ?

In the case $\mathbb{K}=\mathbb{C}$, Chądzyński and Krasiński [2] proved that for a complex polynomial mapping $F:=\left(f_{1}, \ldots, f_{k}\right): \mathbb{C}^{n} \rightarrow \mathbb{C}^{k}, \mathcal{L}_{\infty}(F)$ is attained on the set $\left\{x \in \mathbb{C}^{n} \mid f_{1}(x) \ldots f_{k}(x)=0\right\}$. On the other hand, the following example shows that a real version of this result fails to hold.

EXAMPLE 1.1. Let

$$
F: \mathbb{R}^{2} \rightarrow \mathbb{R}^{2}, \quad(x, y) \mapsto\left(f_{1}(x, y):=(x-y)^{2}, f_{2}(x, y):=(x-y)^{2}+y^{4}\right) .
$$

It is obvious that $\left\{(x, y) \in \mathbb{R}^{2} \mid f_{1}(x, y) f_{2}(x, y)=0\right\}=\{(x, x) \mid x \in \mathbb{R}\}$ and

$$
\|F(x, x)\|=|x|^{4} \text {. }
$$

2000 Mathematics Subject Classification: Primary 14P10.

Key words and phrases: Łojasiewicz exponent at infinity, curve of tangency. 
Moreover, one can show directly that $\mathcal{L}_{\infty}(F)=2$. Hence, $\mathcal{L}_{\infty}(F)$ is not attained on $\left\{(x, y) \in \mathbb{R}^{2} \mid f_{1}(x, y) f_{2}(x, y)=0\right\}$.

In the case $\mathbb{K}=\mathbb{R}$, using polar curves, Gwoździewicz [5] (see also [6]) gave an explicit bound for the Łojasiewicz exponent at infinity of a real polynomial function with compact zero fiber. Moreover, it was shown in [9] that if $f: \mathbb{R}^{2} \rightarrow \mathbb{R}$ is a positive definite polynomial in two real variables (i.e., $f(x)>0$ as $\|x\| \rightarrow \infty)$, then $\mathcal{L}_{\infty}(f)$ is attained on the polar curve of this polynomial. It seems, however, more difficult to obtain similar results in the general case.

Let now $f: \mathbb{R}^{n} \rightarrow \mathbb{R}$ be a nonconstant real polynomial function. The object of this paper is to provide a method to determine $\mathcal{L}_{\infty}(f)$, using the information from the "curve of tangency" (see Section 2 for definition). It is worth noting that, in contrast to [5] and [9], we do not need to assume the compactness of $f^{-1}(0)$.

As an application, we give a computational criterion to decide if the Łojasiewicz exponent at infinity is finite or not. We also obtain a formula to calculate the set of points at which a polynomial $f$ is not proper. This set was introduced and studied by Jelonek in several papers (see [10], [11], for instance); it plays a substantial role in the geometric approach to the Jacobian conjecture.

Moreover, based again on the curve of tangency, some links between the Łojasiewicz exponent at infinity of $f$ and the following interesting problems are established:

- How to tell if the polynomial $f$ is bounded from below or not?

- Suppose that the polynomial $f$ is bounded from below. Find the global infimum

$$
f_{*}:=\inf \left\{f(x) \mid x \in \mathbb{R}^{n}\right\} .
$$

The first problem was originally posed by Shor [17] in his fundamental paper about optimization of real multivariable polynomials. On the other hand, as is well-known, the second problem is NP-hard even when the degree of $f$ is fixed to be 4 [16].

The results obtained by Chądzyński and Krasiński in [2], [3] have played an inspiring role in undertaking this research. On the other hand, the main idea used in our argument is the notion of curve of tangency, which was taken from [7].

The paper is organized as follows. The notion of the curve of tangency is recalled in Section 2. The main result and its proof are given in Section 3. Some conclusions about the Łojasiewicz exponent at infinity are obtained in Section 4 . 
2. The curve of tangency. In this section we briefly recall the notion of the curve of tangency. For details the reader may consult [7] (see also [4]).

Throughout this paper, $f: \mathbb{R}^{n} \rightarrow \mathbb{R}$ is a nonconstant polynomial function, and we shall denote by $\Sigma(f)$ the set of critical points of $f$.

Set

$$
X:=\left\{(x, a) \in \mathbb{R}^{n} \times \mathbb{R}^{n} \mid \operatorname{rank}\left(\begin{array}{ccc}
\partial f / \partial x_{1} & \cdots & \partial f / \partial x_{n} \\
x_{1}-a_{1} & \cdots & x_{n}-a_{n}
\end{array}\right) \leq 1\right\} .
$$

For $a \in \mathbb{R}^{n}$, define

$$
\Gamma(a, f):=\left\{x \in \mathbb{R}^{n} \mid x \notin \Sigma(f) \text { and }(x, a) \in X\right\} .
$$

Geometrically, $\Gamma(a, f)$ consists of all points $x \notin \Sigma(f)$ where the level sets of $f$ are tangent to $\mathbb{S}_{\|x-a\|}(a)$; here $\mathbb{S}_{r}(a):=\left\{x \in \mathbb{R}^{n} \mid\|x-a\|=r\right\}$ denotes the sphere in $\mathbb{R}^{n}$ centered at $a$ with radius $r$. We also write $\mathbb{B}_{r}(a):=\left\{x \in \mathbb{R}^{n} \mid\right.$ $\|x-a\|<r\}$ for the open ball.

The following is a simple fact about the set $\Gamma(a, f)$.

LEMMA 2.1. With the previous notations:

(i) $\Gamma(a, f)$ is a nonempty, unbounded and semi-algebraic set;

(ii) There exists a proper algebraic set $\Omega \subsetneq \mathbb{R}^{n}$ such that $\Gamma(a, f)$ is a one-dimensional submanifold of $\mathbb{R}^{n}$ for each $a \in \mathbb{R}^{n} \backslash \Omega$.

Proof. (i) Clearly, the sets $X$ and $\Sigma(f)$ are algebraic; and hence, by definition, $\Gamma(a, f)$ is semi-algebraic.

To prove that $\Gamma(a, f)$ is nonempty and unbounded, define

$$
\begin{aligned}
& C:=\left\{x \in \mathbb{R}^{n} \mid f(x)=\min \left\{f(y) \mid\|y-a\|=\|x-a\|, y \in \mathbb{R}^{n}\right\}\right\}, \\
& D:=\left\{x \in \mathbb{R}^{n} \mid f(x)=\max \left\{f(y) \mid\|y-a\|=\|x-a\|, y \in \mathbb{R}^{n}\right\}\right\} .
\end{aligned}
$$

The sets $C$ and $D$ are semi-algebraic and obviously unbounded in $\mathbb{R}^{n}$. Moreover, there exists $r>0$ such that either

$$
C \backslash \mathbb{B}_{r}(a) \subset \Gamma(a, f) \text { or } \quad D \backslash \mathbb{B}_{r}(a) \subset \Gamma(a, f) .
$$

Indeed, suppose that this is not the case. Then, by the curve selection lemma at infinity (see [14], [15]), there exist $\delta>0$ and Nash (i.e., analytic algebraic) functions $\varphi:(0, \delta] \rightarrow C$ and $\psi:(0, \delta] \rightarrow D$ such that

- $\lim _{\tau \rightarrow 0}\|\varphi(\tau)\|=\lim _{\tau \rightarrow 0}\|\psi(\tau)\|=\infty$,

- $\varphi(\tau) \notin \Gamma(a, f)$ and $\psi(\tau) \notin \Gamma(a, f)$ for all $\tau \in(0, \delta]$.

According to Lagrange's multiplier theorem, this implies that

$$
\operatorname{grad} f[\varphi(\tau)]=\operatorname{grad} f[\psi(\tau)]=0 \quad \text { for all } \tau \in(0, \delta] .
$$

Consequently, $(f \circ \varphi)^{\prime}$ and $(f \circ \psi)^{\prime}$ vanish in $(0, \delta]$, so that the functions $\tau \mapsto f[\varphi(\tau)]$ and $\tau \mapsto f[\psi(\tau)], \tau \in(0, \delta]$, are constant. Hence $f$ is constant, which is a contradiction. 
(ii) Set $Y:=\Sigma(f) \times \mathbb{R}^{n}$. We shall show that $X \backslash Y$ is a smooth manifold of dimension $n+1$. Indeed, let $\left(x^{0}, a^{0}\right) \in X \backslash Y$. Without loss of generality, we can assume that $\frac{\partial f}{\partial x_{n}}\left(x^{0}\right) \neq 0$. Then there exists a neighborhood $U$ of $x^{0}$ in $\mathbb{R}^{n}$ such that $\frac{\partial f}{\partial x_{n}}(x) \neq 0$ for all $x \in U$. Consequently, we may write

$$
(X \backslash Y) \cap\left(U \times \mathbb{R}^{n}\right)=\left\{(x, a) \in U \times \mathbb{R}^{n} \mid \Phi_{i}(x, a)=0, i=1, \ldots, n-1\right\},
$$

where

$$
\Phi_{i}(x, a):=\left(x_{n}-a_{n}\right) \frac{\partial f}{\partial x_{i}}(x)-\left(x_{i}-a_{i}\right) \frac{\partial f}{\partial x_{n}}(x) .
$$

A direct computation shows that

$$
\operatorname{det}\left(\frac{\partial \Phi_{i}}{\partial a_{j}}(x, a)\right)_{1 \leq i, j \leq n-1}=\left[\frac{\partial f}{\partial x_{n}}(x)\right]^{n-1} \neq 0 \quad \text { for all }(x, a) \in U \times \mathbb{R}^{n} .
$$

Applying the implicit function theorem to the mapping

$$
U \times \mathbb{R}^{n} \rightarrow \mathbb{R}^{n-1}, \quad(x, a) \mapsto\left(\Phi_{1}(x, a), \ldots, \Phi_{n-1}(x, a)\right),
$$

we see that $X \backslash Y$ is an $(n+1)$-dimensional submanifold of $\mathbb{R}^{n} \times \mathbb{R}^{n}$.

We now consider the second projection $\pi_{2}: X \backslash Y \rightarrow \mathbb{R}^{n},(x, a) \mapsto a$. By an algebraic version of Sard's theorem (see [1]), there exists a proper algebraic set $\Omega \subsetneq \mathbb{R}^{n}$ such that for each $a \in \mathbb{R}^{n} \backslash \Omega, \pi_{2}^{-1}(a)$ is a smooth manifold of dimension $(n+1)-n=1$. This implies that $\Gamma(a, f)=\pi_{1}\left(\pi_{2}^{-1}(a)\right)$ is a one-dimensional submanifold of $\mathbb{R}^{n}$, where $\pi_{1}$ is the first projection $X \backslash Y \rightarrow \mathbb{R}^{n},(x, a) \mapsto x$.

This ends the proof.

Definition 2.1 (see [4], [7]). The set $\Gamma(a, f)$, when it is a smooth manifold of dimension 1, will be called the curve of tangency of $f$ with respect to $a \in \mathbb{R}^{n}$.

REMARK 2.1. In [4], [7], the curves of tangency of polynomials are used for different purposes.

3. The main result. In order to formulate the main theorem we shall need some definitions.

Definition 3.1. For any subset $V \subset \mathbb{R}^{n}$, the set $R_{\infty}(f, V)$ of asymptotic values of $f$ on $V$ consists of all $y \in \mathbb{R} \cup\{-\infty,+\infty\}$ for which there exists a sequence $\left\{x^{k}\right\}_{k \in \mathbb{N}} \subset V$ such that $x^{k} \rightarrow \infty$ and $f\left(x^{k}\right) \rightarrow y$.

By a standard argument, based on the curve selection lemma at infinity (see [14], [15]), we have $R_{\infty}(f, \Sigma(f)) \subset f(\Sigma(f))$, the set of critical values of $f$. According to an algebraic version of Sard's theorem (see [1]), this implies that $R_{\infty}(f, \Sigma(f))$ is finite. Moreover, we have

Lemma 3.1. For all $a \in \mathbb{R}^{n}$, the set $R_{\infty}(f, \Gamma(a, f))$ is finite. 
Proof. This follows immediately from [8, Lemma 2.2] (see also [18, Theorem 1.5]).

Furthermore, we can give more concrete information about the set of asymptotic values $R_{\infty}(f, \Gamma(a, f))$ in the case where $\Gamma(a, f)$ is a curve of tangency of $f$. In fact, let $\Omega$ be as in Lemma 2.1. Fix $a \in \mathbb{R}^{n} \backslash \Omega$, so that $\Gamma(a, f)$ is the curve of tangency of $f$. It is not hard to see that for $r>0$ sufficiently large, $\Gamma(a, f) \backslash \mathbb{B}_{r}(a)$ consists of a fixed number of one-dimensional connected components, say $\Gamma_{1}, \ldots, \Gamma_{s}$. Taking $r$ large enough, we infer that, for $i=1, \ldots, s$, there exist $\delta>0$ and a Nash function $\theta_{i}:(0, \delta] \rightarrow \mathbb{R}^{n}$, $\tau \mapsto \theta_{i}(\tau)$, such that $\Gamma_{i}$ is the germ of the curve $x=\theta_{i}(\tau)$ as $\tau \rightarrow 0$. Note that $\theta_{i}$ (or rather its germ at 0 ) is given by a real algebraic Puiseux series in $\tau$. Let

$$
\left\|\theta_{i}(\tau)\right\|:=a_{i} \tau^{\alpha_{i}}+\text { higher order terms in } \tau,
$$

where $a_{i} \in \mathbb{R} \backslash\{0\}$ and $\alpha_{i} \in \mathbb{Q}$. Since $\left\|\theta_{i}(\tau)\right\| \rightarrow+\infty$ as $\tau \rightarrow 0$, we have $\alpha_{i}<0$. We may also assume (taking $\delta>0$ small enough if necessary) that the function $\left\|\theta_{i}\right\|:(0, \delta] \rightarrow \mathbb{R}, \tau \mapsto\left\|\theta_{i}(\tau)\right\|$, is strictly decreasing. Moreover, the function $f \circ \theta_{i}:(0, \delta] \rightarrow \mathbb{R}, \tau \mapsto f\left[\theta_{i}(\tau)\right]$, is strictly increasing, strictly decreasing or constant for $\delta$ small. Hence, it has a $\operatorname{limit}_{i}:=\lim _{\Gamma_{i}} f$ in $\mathbb{R} \cup\{+\infty,-\infty\}$. Furthermore, we also expand

$$
f\left[\theta_{i}(\tau)\right]:=b_{i} \tau^{\beta_{i}}+\text { higher order terms in } \tau, \quad b_{i} \in \mathbb{R} .
$$

If the series $f\left[\theta_{i}(\tau)\right]$ is identically zero, we set $b_{i}=0$ and $\beta_{i}$ arbitrary (unimportant).

Assume that the connected components $\Gamma_{1}, \ldots, \Gamma_{s}$ are numbered in such a way that $t_{1} \leq \cdots \leq t_{s}$. Then we put

$$
I_{1}:=\left\{i \mid t_{i}=t_{1}\right\}, \quad I_{s}:=\left\{i \mid t_{i}=t_{s}\right\} .
$$

There are the following cases to discuss:

(A1) $t_{1}=-\infty$ and $t_{s}=+\infty$.

(A2) $-\infty<t_{1}<0$.

(A3) $0<t_{s}<+\infty$.

(A4) $0 \leq t_{1}, R_{\infty}(f, \Sigma(f)) \neq \emptyset$ and $\min _{t \in R_{\infty}(f, \Sigma(f))} t \leq 0$.

(A5) $t_{s} \leq 0, R_{\infty}(f, \Sigma(f)) \neq \emptyset$ and $\max _{t \in R_{\infty}(f, \Sigma(f))} t \geq 0$.

(A6) $t_{1}=0$ and $\min \left\{b_{i} \mid i \in I_{1}\right\} \leq 0$.

(A7) $t_{s}=0$ and $\max \left\{b_{i} \mid i \in I_{s}\right\} \geq 0$.

(A8) Otherwise.

LEMmA 3.2. The set $f^{-1}(0)$ is not compact if and only if one of Cases (A1)-(A7) holds.

Proof. This is straightforward.

The main result of this paper can now be formulated. 
Theorem 3.1. (Notations as above). If one of Cases (A1)-(A7) holds then $\mathcal{L}_{\infty}(f)=-\infty$; otherwise, there exists a Nash function $\varphi:(0, \varepsilon] \rightarrow \mathbb{R}^{n}$ $(\varepsilon>0)$ with $\lim _{\tau \rightarrow 0}\|\varphi(\tau)\|=\infty$ such that either

(i) $\varphi(\tau) \in \Sigma(f)$ for all $\tau \in(0, \varepsilon]$ and $\mathcal{L}_{\infty}(f)=0$;

(ii) $\varphi(\tau) \in \Gamma(a, f)$ for all $\tau \in(0, \varepsilon]$ and

$$
\mathcal{L}_{\infty}(f)=\min \left\{\beta_{1} / \alpha_{1}, \beta_{s} / \alpha_{s}\right\} .
$$

Proof. By Lemma 3.2, $\mathcal{L}_{\infty}(f)=-\infty$ provided that one of Cases (A1)(A7) holds.

Conversely, suppose that none of Cases (A1)-(A7) occurs, which is equivalent to saying that $f^{-1}(0)$ is compact. This implies that, in expansion (2), $b_{i} \neq 0$ for $i=1, \ldots, s$. Moreover, by (1) and (2), asymptotically as $\tau \rightarrow 0$, we have

$$
\left|f\left[\theta_{i}(\tau)\right]\right| \simeq\left\|\theta_{i}(\tau)\right\|^{\beta_{i} / \alpha_{i}}, \quad i=1, \ldots, s,
$$

where $A \simeq B$ means that $A / B$ lies between two positive constants. Hence, by the definition of $\mathcal{L}_{\infty}(f)$, we get

$$
\mathcal{L}_{\infty}(f) \leq \min _{i=1, \ldots, s} \beta_{i} / \alpha_{i} .
$$

As in the proof of Lemma 2.1 we let

$$
\begin{aligned}
& C:=\left\{x \in \mathbb{R}^{n} \mid f(x)=\min \left\{f(y) \mid\|y-a\|=\|x-a\|, y \in \mathbb{R}^{n}\right\}\right\}, \\
& D:=\left\{x \in \mathbb{R}^{n} \mid f(x)=\max \left\{f(y) \mid\|y-a\|=\|x-a\|, y \in \mathbb{R}^{n}\right\}\right\} .
\end{aligned}
$$

There are three different cases to discuss.

CASE 1: $C \backslash \mathbb{B}_{r}(a) \nsubseteq \Gamma(a, f)$ for all $r>0$. Then there exist $\varepsilon>0$ and a Nash function $\varphi(0, \varepsilon] \rightarrow C$ such that

$$
\lim _{\tau \rightarrow 0}\|\varphi(\tau)\|=\infty, \quad \varphi(\tau) \notin \Gamma(a, f) \quad \text { for all } \tau \in(0, \varepsilon] .
$$

In view of Lagrange's multiplier theorem,

$$
\operatorname{grad} f[\varphi(\tau)]=0 \quad \text { for all } \tau \in(0, \varepsilon] \text {. }
$$

This implies that $\tau \mapsto f[\varphi(\tau)]$ is constant, say $m$, for $\tau$ small. As a corollary, $\mathcal{L}_{\infty}(f) \leq 0$. On the other hand, we have $m>0$ because $f^{-1}(0)$ is compact. Moreover, by definition, $|f(x)|=f(x) \geq m>0$ for $\|x\|$ large enough. This leads to $\mathcal{L}_{\infty}(f) \geq 0$. Therefore $\mathcal{L}_{\infty}(f)=0$.

CASE 2: $D \backslash \mathbb{B}_{r}(a) \nsubseteq \Gamma(a, f)$ for all $r>0$. By entirely analogous arguments to those in Case 1, we also get statement (i).

CASE 3: $C \backslash \mathbb{B}_{r}(a) \subseteq \Gamma(a, f)$ and $D \backslash \mathbb{B}_{r}(a) \subseteq \Gamma(a, f)$ for some $r>0$. Since $f^{-1}(0)$ is compact, $f(x)$ does not change sign for $\|x\|$ sufficiently large. Taking $-f$ instead of $f$ if needed, we can assume that $f(x)>0$ for $\|x\|$ large enough. 
Notice that the set $C$ is semi-algebraic and unbounded in $\mathbb{R}^{n}$. Hence, $C \backslash \mathbb{B}_{r}(a) \subseteq \Gamma(a, f)$ implies that $C \backslash \mathbb{B}_{r}(a)$ must contain the connected component $\Gamma_{1}$, and possibly some other connected components, say $\Gamma_{2}, \ldots, \Gamma_{k}$.

Let $x \in \mathbb{R}^{n}$ with $\|x\| \gg 1$. Since $\lim _{\tau \rightarrow 0}\left\|\theta_{1}(\tau)\right\|=\infty$ and $\tau \mapsto\left\|\theta_{1}(\tau)\right\|$ is strictly decreasing, we have $\left\|\theta_{1}(\tau)-a\right\|=\|x-a\|$ for some $\tau \in(0, \delta]$. Hence,

$$
|f(x)|=f(x) \geq \min \left\{f(y) \mid\|y-a\|=\|x-a\|, y \in \mathbb{R}^{n}\right\}=f\left[\theta_{1}(\tau)\right] .
$$

On the other hand, it follows from (1) and (2) that

$$
f\left[\theta_{1}(\tau)\right] \simeq\left\|\theta_{1}(\tau)-a\right\|^{\beta_{1} / \alpha_{1}}=\|x-a\|^{\beta_{1} / \alpha_{1}} .
$$

Therefore $\mathcal{L}_{\infty}(f) \geq \beta_{1} / \alpha_{1}$. This fact, together with (3), proves (ii), and hence the theorem is proved.

REMARK 3.1. Suppose that the set of critical points of $f$ is compact. Then, by Theorem 3.1, to determine $\mathcal{L}_{\infty}(f)$ it suffices to compute the Puiseux expansions at infinity of the curve of tangency $\Gamma(a, f)$, which can be performed using a version at infinity of Mac-Millan's result in [12] (see also [13]).

EXAmple 3.1. Let $f(x):=\left[\sum_{i=1}^{n} x_{i}\right]^{2}+1$. We can choose $a$ to be the origin in $\mathbb{R}^{n}$. A direct computation shows that

$$
\Sigma(f)=\left\{x \in \mathbb{R}^{n} \mid \sum_{i=1}^{n} x_{i}=0\right\}, \quad \Gamma(a, f)=\left\{x \in \mathbb{R}^{n} \backslash\{0\} \mid x_{1}=\cdots=x_{n}\right\} .
$$

Moreover, $\mathcal{L}_{\infty}(f)(=0)$ is attained on $\Sigma(f)$.

EXAmPle 3.2. Consider the polynomial in three variables

$$
f(x, y, z):=(x y-1)^{2}+x^{2}+(z-1)^{2} .
$$

Clearly, $f^{-1}(0)=\emptyset$. We can choose $a:=(0,0,0) \in \mathbb{R}^{3}$. Then the curve of tangency $\Gamma(a, f)$ is given by the equations

$$
2 z x y^{2}-2 z y+2 x=0 \quad \text { and } \quad 2 z x^{2} y-2 z x-2 z y+2 y=0 .
$$

Using MAPLE we have found that there are ten (real) connected components of the curve of tangency:

$$
\begin{aligned}
& \Gamma_{ \pm 1}: \varphi_{ \pm 1}=\left(s^{-1}+\frac{1}{2} s+\frac{1}{4} s^{3}+\cdots, s+\frac{1}{2} s^{3}+\cdots,-s^{-4}+\frac{1}{2} s^{-2}+\cdots\right), \\
& \Gamma_{ \pm 2}: \varphi_{ \pm 2}=\left(-\frac{1}{2} s+\frac{3}{8} s^{3}+\cdots,-2 s^{-1}-s+\frac{3}{4} s^{3}+\cdots, 1-\frac{17}{4} s^{2}+\cdots\right), \\
& \Gamma_{ \pm 3}: \varphi_{ \pm 3}=\left(\frac{2}{3} s^{-1}+\frac{1}{4} s+\cdots,-\frac{2}{3} s^{-1}+\frac{1}{2} s+\cdots,-\frac{9}{4} s^{2}+\frac{27}{16} s^{4}+\cdots\right), \\
& \Gamma_{ \pm 4}: \varphi_{ \pm 4}=\left(2 s^{-1}-\frac{1}{4} s+\cdots, 2 s^{-1}-\frac{1}{2} s+\cdots,-\frac{1}{4} s^{2}-\frac{3}{16} s^{4}+\cdots\right), \\
& \Gamma_{ \pm 5}: \varphi_{ \pm 5}=\left(0,0, s^{-1}\right) .
\end{aligned}
$$


Here $s \rightarrow \pm 0$. Then substituting these expansions in $f$ we get

$$
\begin{aligned}
& \left.f\right|_{\Gamma_{ \pm 1}}=s^{-8}-s^{-6}+O\left(s^{-4}\right) \\
& \left.f\right|_{\Gamma_{ \pm 2}}=\frac{1}{4} s^{2}+\frac{71}{4} s^{4}+O\left(s^{6}\right), \\
& \left.f\right|_{\Gamma_{ \pm 3}}=\frac{16}{81} s^{-4}+\frac{32}{27} s^{-2}+\frac{20}{9}+\frac{451}{96} s^{2}+O\left(s^{4}\right), \\
& \left.f\right|_{\Gamma_{ \pm 4}}=16 s^{-4}-16 s^{-2}+2+\frac{117}{32} s^{2}+O\left(s^{4}\right), \\
& \left.f\right|_{\Gamma_{ \pm 5}}=2+s^{-2}-2 s^{-1}
\end{aligned}
$$

Consequently,

$$
\begin{array}{ll}
\alpha_{ \pm 1}=-4, & \beta_{ \pm 1}=-8, \\
\alpha_{ \pm 2}=-1, & \beta_{ \pm 2}=2, \\
\alpha_{ \pm 3}=-1, & \beta_{ \pm 3}=-4, \\
\alpha_{ \pm 4}=-1, & \beta_{ \pm 4}=-4, \\
\alpha_{ \pm 5}=-1, & \beta_{ \pm 5}=-2 .
\end{array}
$$

Hence,

$$
\mathcal{L}_{\infty}(f)=\min \left\{\frac{-8}{-4}, \frac{2}{-1}, \frac{-4}{-1}, \frac{-4}{-1}, \frac{-2}{-1}\right\}=-2 .
$$

4. Corollaries. Let us keep the notations of Section 3. We now give some applications of Theorem 3.1. The easiest consequence is an answer to the question of [17]:

Corollary 4.1.

(i) $f$ is bounded from below if and only if $t_{1}>-\infty$.

(ii) $f$ is bounded from above if and only if $t_{s}<+\infty$.

Proof. This follows immediately from the proofs of Lemma 2.1 and Theorem 3.1.

Next we put

$$
\begin{aligned}
& t_{*}:= \begin{cases}t_{1} & \text { if } R_{\infty}(f, \Sigma(f))=\emptyset, \\
\min \left\{t_{1}, \min _{t \in R_{\infty}(f, \Sigma(f))} t\right\} & \text { if } R_{\infty}(f, \Sigma(f)) \neq \emptyset,\end{cases} \\
& t^{*}:= \begin{cases}t_{s} & \text { if } R_{\infty}(f, \Sigma(f))=\emptyset, \\
\max \left\{t_{s}, \max _{t \in R_{\infty}(f, \Sigma(f))} t\right\} & \text { if } R_{\infty}(f, \Sigma(f)) \neq \emptyset .\end{cases}
\end{aligned}
$$

Recall that the polynomial $f: \mathbb{R}^{n} \rightarrow \mathbb{R}$ is not proper at a point $t \in \mathbb{R}$ if there is no neighborhood $U$ of $t$ such that $f^{-1}(\bar{U})$ is compact. In other words, $f$ is not proper at $t$ if there is a sequence $\left\{x^{k}\right\}_{k \in \mathbb{N}} \subset \mathbb{R}^{n}$ such that $x^{k} \rightarrow \infty$ and $f\left(x^{k}\right) \rightarrow t$, i.e., $t \in \mathbb{R} \cap R_{\infty}\left(f, \mathbb{R}^{n}\right)$. The polynomial $f$ is called proper if $\mathbb{R} \cap R_{\infty}\left(f, \mathbb{R}^{n}\right)=\emptyset$. 
For each $t \in \mathbb{R}$ we will denote by $f-t$ the polynomial function $\mathbb{R}^{n} \rightarrow \mathbb{R}$, $x \mapsto f(x)-t$.

COROllary 4.2. With the previous notations:

(i) For each $t \in\left(t_{*}, t^{*}\right)$ we have

$$
\mathcal{L}_{\infty}(f-t)=-\infty .
$$

(ii) $f$ is bounded neither from below nor from above if and only if

$$
\mathcal{L}_{\infty}(f-t)=-\infty \quad \text { for all } t \in \mathbb{R} .
$$

(iii) $f$ is proper if and only if $\mathcal{L}_{\infty}(f-t)$ is a positive constant for all $t \in \mathbb{R}$.

(iv) Suppose that $f$ is not proper.

(iv-1) If $t_{*}>-\infty$, then

$$
\mathcal{L}_{\infty}(f-t)=0 \quad \text { if and only if } t<t_{*} .
$$

(iv-2) If $t^{*}<+\infty$, then

$$
\mathcal{L}_{\infty}(f-t)=0 \text { if and only if } t>t^{*} .
$$

Proof. (i) It follows from the definitions that $f^{-1}(t)$ is not compact for all $t \in\left(t_{*}, t^{*}\right)$.

(ii) By Corollary 4.1, $f$ is bounded neither from below nor from above if and only if $t_{1}=-\infty$ and $t_{s}=+\infty$, which is equivalent to $f^{-1}(t)$ being noncompact for all $t \in \mathbb{R}$. This proves (ii).

(iii) If $\mathcal{L}_{\infty}(f)>0$, then obviously $f$ is proper. Conversely, suppose that $f$ is proper. Then $f^{-1}(0)$ is compact. Moreover, in (2), $b_{i} \neq 0$ and the exponent $\beta_{i}, i=1, \ldots, s$, must be negative. But $\alpha_{i}<0$ for $i=1, \ldots, s$. Hence, by Theorem 3.1,

$$
\mathcal{L}_{\infty}(f)=\min \left\{\frac{\beta_{1}}{\alpha_{1}}, \frac{\beta_{s}}{\alpha_{s}}\right\}>0 .
$$

On the other hand, as expressed by the notation $\Gamma(a, f)$, the polynomials $f$ with the same gradient $\nabla f$ have the same tangency variety, in other words,

$$
\Gamma(a, f-t)=\Gamma(a, f) \quad \text { for all } t \in \mathbb{R} .
$$

Furthermore, it is worth noting that $f$ is proper if and only if $f-t$ is proper for each $t \in \mathbb{R}$. Therefore $\mathcal{L}_{\infty}(f-t)=\mathcal{L}_{\infty}(f)>0$, which proves (iii).

(iv) We only prove (iv-1); the proof of (iv-2) uses entirely analogous arguments. So suppose that $f$ is not proper and $t_{*}>-\infty$. Hence, in particular, $t^{*}=t_{s}=+\infty$. Then one can easily see that $t_{1}<+\infty$, and hence $\beta_{1} \geq 0$. There are two cases to consider. 
CASE 1: $R_{\infty}(f, \Sigma(f))=\emptyset$. If the series $f\left[\theta_{i}(\tau)\right]-t_{1}$ is identically zero for some $i \in I_{1}=\left\{i \mid t_{i}=t_{1}\right\}$, then it is clear that

$$
\mathcal{L}_{\infty}(f-t)= \begin{cases}-\infty & \text { if } t \geq t_{1} \\ 0 & \text { if } t<t_{1} .\end{cases}
$$

Now suppose that $f\left[\theta_{i}(\tau)\right]-t_{1} \not \equiv 0$ for all $i \in I_{1}$. Then it is not difficult to see that the proof of Theorem 3.1 also shows that $\mathcal{L}_{\infty}\left(f-t_{1}\right)<0$ and $\mathcal{L}_{\infty}(f-t)=0$ for all $t<t_{1}$. On the other hand, by $(\mathrm{i}), \mathcal{L}_{\infty}(f-t)=-\infty$ for all $t>t_{1}$. This proves (iv-1) in Case 1 .

CASE 2: $R_{\infty}(f, \Sigma(f)) \neq \emptyset$. Then it is not hard to verify that

$$
\mathcal{L}_{\infty}(f-t)= \begin{cases}0 & \text { if } t<t_{*}, \\ -\infty & \text { if } t>t_{*}, \\ -\infty & \text { if } t=t_{*} \text { and }\left\{f=t_{*}\right\} \text { is not compact } \\ l & \text { if } t=t_{*} \text { and }\left\{f=t_{*}\right\} \text { is compact }\end{cases}
$$

where $l$ is a negative rational number. As a corollary, we get (iv-1) in Case 2.

From Corollary 4.2, we immediately obtain

Corollary 4.3. Let $f: \mathbb{R}^{n} \rightarrow \mathbb{R}$ be a polynomial function. Then either

(i) the function $\mathbb{R} \rightarrow \mathbb{Q} \cup\{-\infty\}, t \mapsto \mathcal{L}_{\infty}(f-t)$, is constant, or

(ii) there exists a stratification

$$
\mathbb{R}=(-\infty, \lambda) \cup\{\lambda\} \cup(\lambda,+\infty)
$$

such that the function $t \mapsto \mathcal{L}_{\infty}(f-t)$ is constant on each stratum.

Proof. Suppose that (i) does not hold. Let

$$
\lambda:= \begin{cases}t_{*} & \text { if } t_{*}>-\infty \\ t^{*} & \text { if } t^{*}<+\infty\end{cases}
$$

Then (ii) follows from Corollary 4.2.

The following corollary says that the set of points at which the polynomial $f$ is not proper can be computed using the information from the curve of tangency and the Łojasiewicz exponent at infinity.

Corollary 4.4. We have

$$
\mathbb{R} \cap R_{\infty}\left(f, \mathbb{R}^{n}\right)= \begin{cases}\emptyset & \text { if } t_{*}=t^{*}, \\ \mathbb{R} & \text { if } t_{*}=-\infty \text { and } t^{*}=+\infty \\ \left\{t \in \mathbb{R} \mid \mathcal{L}_{\infty}(f-t)<0\right\} & \text { otherwise. }\end{cases}
$$

Proof. The statement follows directly from Corollary 4.2.

We now suppose that the polynomial $f: \mathbb{R}^{n} \rightarrow \mathbb{R}$ is bounded from below and consider the global optimization problem:

$$
f_{*}:=\inf \left\{f(x) \mid x \in \mathbb{R}^{n}\right\} .
$$


As is well known, if $f$ attains a minimum in $x^{*} \in \mathbb{R}^{n}$, i.e., $f\left(x^{*}\right) \leq f(x)$ for all $x \in \mathbb{R}^{n}$, then the gradient of $f$ vanishes at $x^{*}$; in other words, $f_{*}=f\left(x^{*}\right)$ is a critical value of $f$. On the other hand, there are polynomials that are bounded from below on $\mathbb{R}^{n}$ and yet do not attain a minimum on $\mathbb{R}^{n}$. In such cases, the following result shows that the global infimum is characterized in terms of the Łojasiewicz exponent at infinity.

Corollary 4.5. Suppose that the polynomial $f: \mathbb{R}^{n} \rightarrow \mathbb{R}$ is bounded from below. If $f$ does not attain its infimum $f_{*}$, then $\mathcal{L}_{\infty}\left(f-f_{*}\right)$ is a negative (finite) number, and moreover

$$
\mathcal{L}_{\infty}(f-t)= \begin{cases}0 & \text { if } t<f_{*}, \\ -\infty & \text { if } t>f_{*} .\end{cases}
$$

Proof. Indeed, it is not difficult to see that $f_{*}=t_{1}$. Then the statement follows from Corollary 4.2.

Let now $F:=\left(f_{1}, \ldots, f_{k}\right): \mathbb{R}^{n} \rightarrow \mathbb{R}^{k}$ be a polynomial mapping. Notice that the Eojasiewicz inequality does not depend on a particular norm in $\mathbb{R}^{n}$, so we shall use the Euclidian norm $\|\cdot\|$. Then consider the polynomial function $\|F\|^{2}: \mathbb{R}^{n} \rightarrow \mathbb{R}, x \mapsto\|F(x)\|^{2}$. By definition, one can easily see that

$$
\mathcal{L}_{\infty}(F)=\frac{1}{2} \mathcal{L}_{\infty}\left(\|F\|^{2}\right) .
$$

Hence, directly from Theorem 3.1 we get

Corollary 4.6. If $F^{-1}(0)$ is not compact, then $\mathcal{L}_{\infty}(F)=-\infty$; otherwise, there exist $\varepsilon>0$ and a Nash function

$$
\varphi:(0, \varepsilon] \rightarrow \Sigma\left(\|F\|^{2}\right) \cup \Gamma\left(a,\|F\|^{2}\right), \quad \tau \mapsto \varphi(\tau),
$$

such that $\lim _{\tau \rightarrow 0}\|\varphi(\tau)\|=\infty$ and

$$
\mathcal{L}_{\infty}(F)=\frac{\operatorname{val}(\|F[\varphi(\tau)]\|)}{\operatorname{val}(\|\varphi(\tau)\|)},
$$

where $\operatorname{val}(\cdot)$ denotes the natural valuation of series with respect to $\tau$; in particular, the number $\mathcal{L}_{\infty}(F)$ is rational.

REMARK 4.1. By entirely analogous arguments but working in a small sphere instead of in the complement of a large sphere, it is not hard to obtain similar results for the local Łojasiewicz exponent of real analytic mapping germs. We leave the details to the reader.

Acknowledgments. We thank the referee for pointing out an error in an earlier version of this paper. The second author thanks the Abdus Salam International Centre for Theoretical Physics, Trieste, Italy, where this paper was written, for hospitality and support. 


\section{References}

[1] R. Benedetti and J.-J. Risler, Real Algebraic and Semi-Algebraic Sets, Actualités Math., Hermann, 1990.

[2] J. Chądzyński and T. Krasiński, A set on which the Eojasiewicz exponent at infinity is attained, Ann. Polon. Math. 67 (1997), 191-197.

[3] - - - A set on which the local Eojasiewicz exponent is attained, ibid., 297-301.

[4] A. H. Durfee, The index of grad $f(x, y)$, Topology 37 (1998), 1339-1361.

[5] J. Gwoździewicz, Growth at infinity of a polynomial with a compact zero set, in: Singularities Symposium- - oojasiewicz 70, Banach Center Publ. 44, Inst. Math., Polish Acad. Sci., 1998, 123-128.

[6] - , The Eojasiewicz exponent of an analytic function at an isolated zero, Comment. Math. Helv. 74 (1999), 364-375.

[7] Hà Huy Vui and Nguyen Thi Thào, On the topology of real polynomials, in preparation.

[8] Hà Huy Vui and Phạm Tiến Sơn, Global optimization of polynomials using the truncated tangency variety and sums of squares, SIAM J. Optim. 19 (2008), 941951.

[9] P. Jankowski, The Eojasiewicz exponent at infinity of a polynomial of two real variables, Bull. Polish Acad. Sci. Math. 50 (2002), 25-31.

[10] Z. Jelonek, The set of points at which a polynomial map is not proper, Ann. Polon. Math. 58 (1993), 259-266.

[11] - , Testing sets for properness of polynomial mappings, Math. Ann. 315 (1999), $1-35$.

[12] W. D. Mac-Millan, A method for determining the solutions of a system of analytic functions in the neighborhood of a branch point, Math. Ann. 72 (1912), 180-202.

[13] J. Maurer, Puiseux expansion for space curves, Manuscripta Math. 32 (1980), 91100.

[14] J. Milnor, Singular Points of Complex Hypersurfaces, Ann. of Math. Stud. 61, Princeton Univ. Press, 1968.

[15] A. Némethi and A. Zaharia, Milnor fibration at infinity, Indag. Math. 3 (1992), 323-335.

[16] Y. Nesterov, Squared functional systems and optimization problems, in: High Performance Optimization, H. Frenk et al. (eds.), Kluwer, 2000, 405-440.

[17] N. Z. Shor, Class of global minimum bounds of polynomial functions, Cybernetics 23 (1987), 731-734.

[18] Tạ Lê Loi and A. Zaharia, Bifurcation sets of functions definable in o-minimal structures, Illinois J. Math. 42 (1998), 449-457.

Institute of Mathematics

18, Hoang Quoc Viet Road

Cau Giay District 10307

Hanoi, Vietnam

E-mail: hhvui@math.ac.vn
Department of Mathematics

University of Dalat

Dalat, Vietnam

E-mail: pham_ts@yahoo.co.uk

Received 24.7.2007

and in final form 25.6.2008 